\title{
ANALYSIS OF MULTI-USER CONGESTION CONTROL FOR VIDEO STREAMING OVER WIRELESS NETWORKS
}

\author{
Xiaoqing Zhu and Bernd Girod \\ Information Systems Laboratory, Stanford University, CA 94305, U.S.A. \\ \{zhuxq,bgirod\}@stanford.edu \\ Invited Paper
}

\begin{abstract}
When multiple video sources are live-encoded and transmitted over a common wireless network, each stream needs to adapt its encoding parameters to wireless channel fluctuations, so as to avoid congesting the network. We present a stochastic system model for analyzing multi-user congestion control for live video coding and streaming over a wireless network. Variations in video content complexities and wireless channel conditions are modeled as independent Markov processes, which jointly determine the bottleneck queue size of each stream. Interaction among multiple users are captured by a simple model of random traffic contention. Using the model, we investigate two distributed congestion control policies: an approach based on stochastic dynamic programming (SDP) and a greedy heuristic. Compared to fixed-quality coding with no congestion control, performance gains in the range of $0.5-1.3 \mathrm{~dB}$ in average video quality are reported for the optimized schemes from simulation results.
\end{abstract}

\section{INTRODUCTION}

The advance of wireless networking technologies and the availability of low-cost video cameras are compelling for many applications, ranging from surveillance camera networks to visual communication in search-and-rescue operations. In such scenarios, multiple video streams are captured at different locations, and need to be live-encoded and transmitted over the network with low latency.

The wireless channel fluctuation and temporal variation of video content, however, typically introduce queuing delays. Moreover, multiple simultaneous video streams can easily congest a shared wireless network. It is therefore important for each user to perform congestion control by adapting its encoding parameters to video and channel variations and striving for the best tradeoff between network congestion and video distortion.

We present a stochastic model for such systems, and formulate the congestion control problem in the context of controlled Markov decision chain [1]. The system evolution is expressed as the transitional probability of system states, including video complexity, channel condition and bottleneck queue size of each stream, given a permissable action,

This work is partially supported by NSF Grant CCR0325639 namely the choice of encoder quantization step size. Interaction among multiple users is captured by a probabilistic model for traffic contention among nodes with non-empty queues. The expected performance of a congestion control policy can then be evaluated based on the equilibrium state distribution of the system. To minimize the expected network congestion and video distortion of all streams, a distributed congestion control policy is derived by performing stochastic dynamic programming (SDP) at each user. A greedy heuristic is also proposed to achieve most of the performance gain with reduced computational complexity.

After a brief survey of related work in Section 2, we explain the system model in Section 3 and discuss both the distributed SDP and the heuristic schemes for congestion control in Section 4. In Section 5, the performance of both schemes are compared against fixed-quality coding under simulated scenarios with various video and channel conditions.

\section{RELATED WORK}

Research on congestion control for live video coding over wireless networks can be traced back to rate control schemes in which the encoder adjusts the quantization step size on a per-frame or per-macroblock basis to meet a target average rate. Many rate control algorithms have been proposed for different video coding standards, such as TM5 for MPEG-2 [2] and [3] for H.264/AVC. We, instead, seek to optimize the tradeoff between network congestion and video distortion as in [4], for multi-user video streaming over wireless networks.

Formulation of the rate control problem over a timevarying wireless channel has been studied in [5], where the authors also propose a optimal solution based on stochastic dynamic programming. In [6], the SDP approach is applied to live video streaming, where the sender prunes the preencoded video packets and the receiver adjusts the playout deadline in a jointly optimized fashion. The problem formulation in both works for the single-stream case has inspired our investigation into the multi-stream scenario, for which we further introduce a probabilistic model to capture the traffic contention among multiple streams. 




Fig. 1. System diagram for multi-user video streaming over wireless network. Each encoder performs congestion control by adjusting the quantization step size $Q_{s}$. End-toend delay is dominated by the bottleneck backlog $L_{s}$.

\section{SYSTEM MODEL}

Consider $S$ simultaneous video streams over a wireless network. Transmission opportunities are slotted into durations of $\Delta T$, and the frame arrival interval is $T_{f}=M \Delta T$. Each user sends a sequence of frames with complexity $X_{s, n}$ over a given route. The encoded frame size $B_{s, n}$ and distortion $D_{s, n}$ are controlled by the encoder quantization step size $Q_{s, n}$. The end-to-end delay $T_{s, n}$ for delivering each frame is assumed to be dominated by the bottleneck node on the path, with transmission rate $C_{s, t}$ and backlog size $L_{s, t}$ at each time slot $t$. The various system states are depicted in Fig. 1. System evolution as a stochastic process is explained in the following subsections.

\subsection{Video content variation}

We characterize video content complexity $X_{s, n}$ of each frame by its mean absolute difference (MAD) per pixel with respect to the previous frame. Its evolution is modeled as a stationary first-order Gauss-Markov process with mean $\mu_{x}$ and variance $\sigma_{x}{ }^{2}$ :

$$
X_{s, n+1}-\mu_{x}=a\left(X_{s, n}-\mu_{x}\right)+N_{s, n},
$$

where $N_{s, n} \sim \mathcal{N}\left(0,\left(1-a^{2}\right){\sigma_{x}}^{2}\right)$.

Following observations in [7], the encoded frame size and distortion given a quantization step size $Q$ can be approximated as:

$$
\begin{aligned}
& B_{s, n}=f_{B}\left(X_{s, n}, Q\right)=\alpha_{s} \frac{X_{s, n}}{Q} \\
& D_{s, n}=f_{D}\left(X_{s, n}, Q\right)=\beta_{s} Q .
\end{aligned}
$$

The parameters $\alpha_{s}$ and $\beta_{s}$ need to be fitted with empirical data from actual encodings for each video stream.

The trace of video content complexity as well as encoder parameter fit is plotted in Fig. 2 for the two video sequences used in the simulations in Section 5. As shown in the figures, the average rate and distortion predicted by the model closely match the experimental results.

\subsection{Wireless channel fluctuation}

Fluctuation of the wireless channel condition is typically modeled as a Markov chain, due to effects such as multi-
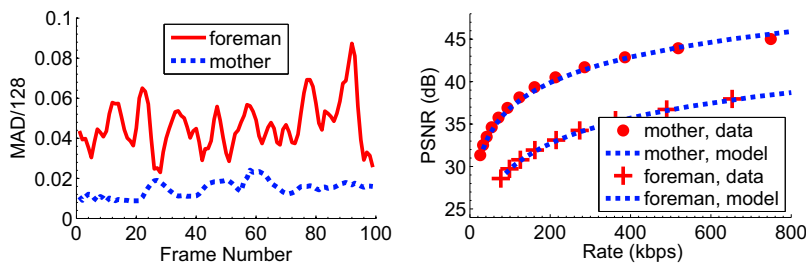

Fig. 2. Trace of video content complexity (left) and fit of encoder rate-distortion performance (right) for CIF video sequences Foreman and Mother and Daughter

path fading and shadowing [8][9]. The time-varying signalto-interference/noise ratio (SINR) usually affects bit-errorrate observed on the channel, which in turn leads to varying effective throughput, as a result of channel coding and retransmission. In this work, the wireless channel on each path is modeled as error-free, with time-varying throughput $\mathbf{C}$, specified by state transitional probabilities

$P_{t+1 \mid t}(\mathbf{C} \mid \mathbf{c})^{1}$ and average channel rates in each state. Note that wireless nodes close to each other typically experience similar channel conditions. The correlation coefficients depend on their physical locations and the processes need to be jointly modeled. For simplicity, we only consider the special case where all bottleneck nodes observe the same channel condition, and henceforth drop the $s$ indices while denoting the channel rates. The analysis and optimization, on the other hand, can extend to the more general case.

\subsection{Bottleneck backlog}

Given current queue size $L_{s, t}$ and observed channel rate $C_{t}$ at the bottleneck, the backlog in the next time slot $L_{s, t+1}$ can be predicted as follows:

$$
L_{s, t+1}= \begin{cases}\max \left\{L_{s, t}+B_{s, t}^{\prime}-C_{t} \Delta T, 0\right\} & w \cdot p \cdot \frac{1}{S^{\prime}} \\ L_{s, t}+B_{s, t}^{\prime} & w \cdot p \cdot 1-\frac{1}{S^{\prime}} .\end{cases}
$$

In (4), $B_{s, t}^{\prime}$ corresponds to the encoded frame size $B_{s, n}$ at times when a new frame arrives:

$$
B_{s, t}^{\prime}= \begin{cases}B_{s, n}=f_{B}\left(X_{s, n}, Q_{s, n}\right) & t=n M \\ 0 & \bmod (t, M) \neq 0\end{cases}
$$

and $S^{\prime}$ denotes the number of bottleneck nodes with nonempty backlogs competing with equal opportunities for wireless media access. This simple probabilistic model is motivated by the CSMA/CA media access control protocols used by wireless LAN cards following the IEEE 802.11 standard, where nodes with data for transmission compete for channel access following the same random backoff procedure.

\footnotetext{
${ }^{1}$ We denote random variables in capital letters and their previous-time-slot realizations in the conditional probabilities in lowercase. Vectors in boldface refer to the collection over all $S$ streams.
} 


\subsection{System evolution}

Combining the model components together, evolution of the entire system state space can be expressed as:

$$
\begin{gathered}
P_{t+1 \mid t}(\mathbf{X}, C, \mathbf{L} \mid \mathbf{x}, c, \mathbf{l}, \mathbf{q})=\prod_{s=1}^{S}\left(P_{t+1 \mid t}\left(X_{s} \mid x_{s}\right) P_{t+1 \mid t}(C \mid c)\right. \\
P_{t+1 \mid t}(\mathbf{L} \mid \mathbf{l}, c, \mathbf{x}, \mathbf{q}),
\end{gathered}
$$

given current system states $\mathbf{x}, c, \mathbf{l}$ and action $\mathbf{q}$.

In (6), the decomposition of the joint state transitional probability assumes statistical independence between video content of different streams and the wireless channel condition. The conditional probability for $X_{s}$ can be calculated from (1) and the evolution of $C$ is specified in Subsection 3.2. The last term $P_{t+1 \mid t}(\mathbf{L})$ is computed according to (2), (4) and (5).

\section{CONGESTION CONTROL POLICIES}

Given current system state, the encoder quantization step size is chosen according to the control policy $\mathbf{Q}=f_{Q}(\mathbf{X}, C, \mathbf{L})$. This in turn determines the state transitional probability by substituting $f_{Q}($.$) for \mathbf{Q}$ into (6). The equilibrium state distribution $\mathcal{P}$ can then be calculated as the Perron-Frobenius eigenvector of the state transition matrix [1].

We propose to optimize both metrics of distortion and network congestion in a lagrangian formulation:

$$
\min _{f_{Q}(.)} \sum_{s} \mathbf{E} D_{s}+\lambda \sum_{s} \mathbf{E} B_{s} \mathbf{E} T_{s},
$$

where network congestion in the second term is the sum of expected delay of each stream weighted by its own traffic rate. The expected video distortion $\mathbf{E} D_{s}$, rate $\mathbf{E} B_{s}$ and delay $\mathbf{E} T_{s}$ are calculated according to the equilibrium state distribution $\mathcal{P}$.

\subsection{Distributed SDP}

There remains the task of finding the optimal control policy for (7). Full search over the product space of all possible system states and candidate policies would be computationally prohibitive. Furthermore, knowledge of the system states such as video complexity and bottleneck queue size of other users may simply not be available in practice. Alternatively, one can approximate (7) by minimizing a finite-horizon cost function at each user:

$$
\min _{q_{s, n}: n=1, \ldots N^{\prime}} J=\frac{1}{N^{\prime}} \sum_{n=1}^{N^{\prime}}\left(d_{s, n}+\lambda b_{s, n} t_{s, n}\right),
$$

This can be solved by recursively minimizing the cost given local system state at each frame interval:

$$
\begin{aligned}
V_{n}\left(x_{s}, c_{s}, l_{s}\right) & =\min _{q_{n}^{\prime}: n^{\prime}=n \ldots N^{\prime}} \sum_{n^{\prime}=n}^{N^{\prime}} d_{s, n^{\prime}}+\lambda b_{s, n^{\prime}} t_{s, n^{\prime}} \\
& =\min _{q_{n}}\left(d_{s, n}+\lambda b_{s, n} t_{s, n}+\mathbf{E} V_{n+1}\right) .
\end{aligned}
$$

Here, the estimated delay for frame $n$ of the $s^{\text {th }}$ stream is $t_{s, n}=\left(b_{s, n}+l_{s, n}\right) S / c_{n}$, assuming equal channel access among all $S$ streams on average. Note that in (9), the choice of the quantization step size is determined not only by the tradeoff between distortion and expected congestion of the current frame, but also influenced by its impact on the expected future cost.

\subsection{Heuristic scheme}

To further reduce computational complexity, one can use a greedy heuristic that ignores the impact on the future:

$$
q_{s, n}^{*}=\arg \min _{q}\left(d_{s, n}+\lambda b_{s, n} t_{s, n}\right) .
$$

In this case, the optimal $q_{s, n}^{*}$ only depends on observations of current frame complexity $x_{s, n}$, encoder RD performance $\alpha_{s}$ and $\beta_{s}$, current channel state $c_{n}$ and bottleneck queue size $l_{s, n}$. It should be noted that the dependency of $q_{s, n}^{*}$ on $l_{s, n}$ is a merit of the chosen optimization objective, since it incorporates the impact of past decisions into the current choice. An alternative lagrangian formulation involving only expected delay and distortion as in $D+\lambda T$, for instance, would have inappropriately ignored the influence of bottleneck queue size $l_{s, n}$ on the current choice of $q_{s, n}$.

\section{SIMULATION RESULTS}

The proposed system is simulated under various scenarios combining different video and channel characteristics. The wireless channel fluctuates between a good $(G)$ and a fading $(F)$ state, each with a random throughput of average rate $C_{G}$ or $C_{F}$. Average state duration is 1.3 seconds for both. As in Fig. 2, video model parameters are fitted with actual data from encoding the Foreman and Mother and Daughter CIF sequences with the H.264/AVC codec [10]. We evaluate the congestion-distortion tradeoff of both the distributed SDP scheme and the greedy heuristic by varying the value of $\lambda$ in the optimization objectives. As a basis for comparison, results from a fix-quality scheme are also included, where the videos are encoded with fixed quantization step sizes without congestion control.

Figure 3 compares the delay-quality tradeoff of the three schemes for streaming only Foreman or Mother and Daughter over the wireless channel. The proposed heuristic and SDP schemes outperform the fix-quality scheme by $0.5-1.0$ $\mathrm{dB}$ in PSNR of video quality given the same average delay. Additional gain up to $0.8-1.3 \mathrm{~dB}$ is achieved by the SDP scheme. The improvement using SDP is more pronounced in the case of Foreman, where both video and channel variations are higher.

When both video streams are transmitted simultaneously over a common network, the relative performance of the three schemes is shown in Fig. 4. The PSNR values correspond to the average video distortion of both streams, and


Fig. 3. Delay-PSNR tradeoff when transmitting only Foreman (left) or Mother and Daughter (right) over a wireless channel. For Foreman, $C_{G}=900 \mathrm{kbps}, C_{F}=300 \mathrm{kbps}$, for Mother and Daughter, $C_{G}=600 \mathrm{kbps}, C_{F}=200 \mathrm{kbps}$. 




Fig. 4. Tradeoff between overall video quality and network congestion with two streams over the network. $C_{G}=900$ kbps, $C_{F}=500 \mathrm{kbps}$.

the overall network congestion is measured as average delay weighted by traffic rate of each stream. For the fix-quality scheme, the different data points correspond to different pairs of quantization step sizes used for the two streams. The best possible performance is represented by the convex hull plotted in dotted line. By adjusting quantization step sizes according to current observations of video complexity, channel rate and backlog size, the heuristic scheme achieves superior video quality by up to $0.8 \mathrm{~dB}$ over fix-quality coding. Additional improvement of up to $0.5 \mathrm{~dB}$ is achieved by the distributed SDP scheme, within a limited range of network congestion. It is also observed in the experiments that the SDP scheme tends to be more conservative, yielding lower video quality and delay than the heuristic given the same $\lambda$.

Figure 5 shows the tradeoff between video quality and delay experienced by each stream, when both are accessing the common wireless media. For the fix-quality scheme, only results using equal quantization step sizes in both streams are displayed. Due to the higher scene complexity, the Foreman sequence experiences higher average delay and lower video quality, when competing over the same wireless media with Mother and Daughter, a sequence that contains slower motion and can be encoded more efficiently. By congestion control, the proposed SDP and heuristic schemes can improve the delay-quality tradeoff for both streams simultaneously.

\section{CONCLUSIONS}

We analyze multi-user congestion control schemes for live video coding and streaming over wireless networks based on a stochastic system model. The model incorporates the impact of video and channel variation, as well as multi-stream traffic contention, into the evaluation of different congestion control policies. A distributed scheme based on stochastic dynamic programming and a greedy heuristic are proposed to minimize the tradeoff between average video distortion and network congestion. Simulation results under various video and channel conditions show gains ranging 0.5-1.3 dB in average video quality achieved by the distributed SDP and heuristic schemes over fix-quality coding.

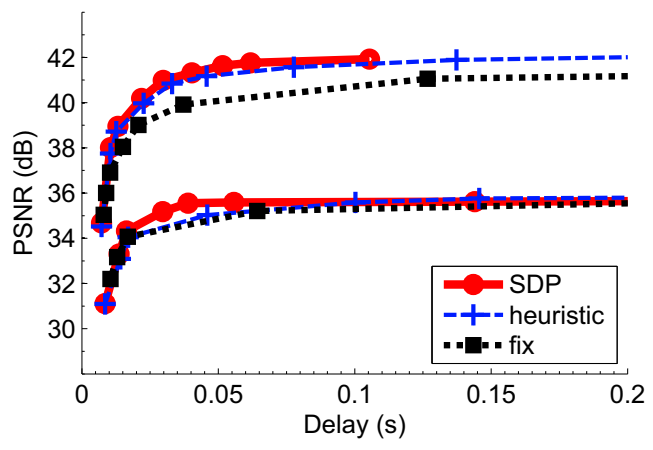

Fig. 5. Delay-PSNR tradeoff of each user.

\section{REFERENCES}

[1] D. Bertsekas, Dynamic Programming and Optimal Control, Vol I., Athena Scientific, Belmont, Massachusetts, USA, 2000.

[2] ISO/IEC/JTC1SC29WG11, MPEG 2 Test Model 5, Rev. 2, Section 10: Rate Control and Quantization Optimization, Apr. 2003.

[3] S. Ma, W. Gao, P. Gao, and Y. Lu, "Rate control for advance video coding (AVC) standard," Proc. International Symposium on Circuits and Systems, Bankok, Thailand, vol. 2, pp. 892-895, May 2003.

[4] X. Zhu, E. Setton, and B. Girod, "Content-adaptive coding and delay-aware rate control for a multi-camera wireless surveillance network," Proc. IEEE International Workshop on Multimedia Signal Processing, Shanghai, China, Oct. 2005.

[5] J. Cabrera and A. Ortega, "Stochastic rate control of video coders for wireless channels," IEEE Trans. on Circuits and Systems for Video Technology, vol. 12, no. 6, pp. 496-510, June 2002.

[6] Y. Li, A. Markopoulou, J. Apostolopoulos, and N. Bambos, "Packet transmission and contentdependent playout for video streaming over wireless networks," Proc. IEEE International Workshop on Multimedia Signal Processing, Shanghai, China, Oct. 2005.

[7] S. Takamura and N. Kobayashi, "MPEG-2 one-pass variable bit rate control algorithm and its LSI implementation," Proc. IEEE International Conference on Image Processing, Thessaloniki, Greece, vol. 1, pp. 942-945, Oct. 2001.

[8] E. N. Gilbert, "Capacity of a burst-noise channel," Bell Syst. Tech. J., vol. 39, no. 9, pp. 1253-1265, 1960.

[9] Q. Zhang and S. A. Kassam, "Finite-state Markov model Rayleigh fading channels," IEEE Trans. on Communications, vol. 47, no. 11, pp. 1688-1692, Nov. 1999.

[10] ITU-T and ISO/IEC JTC 1, Advanced Video Coding for Generic Audiovisual services, ITU-T Recommendation H.264 - ISO/IEC 14496-10(AVC), 2003. 